\title{
Research on healthy catering recommendation space
}

\author{
Yongjie Deng, Yong Liu* and Dongping Tang \\ College of business administration, South China University of Technology, Guangzhou, China
}

Keywords: Healthy catering, Recommended space, TCM dietetic therapy.

\begin{abstract}
This paper conducts an in-depth study on the catering recommendation space based on different situations. By consulting the literature, visiting relevant experts, condensing the theoretical knowledge of TCM (Traditional Chinese Medicine) constitutional dietotherapy theory, it has extracted some key factors that can be effectively utilized in catering theory. The variable framework of the situational catering recommendation system is preliminarily constructed. Also, it has designed a mixed catering recommendation space that combines TCM Constitutional Theory and user situation, which not only meets individual preferences but also improves the users' sub-health status. Finally, through the questionnaire survey, the importance of each attribute in the eyes of consumers has been recognized; then this essay provides a guidance for the recommendation ranking method in the design of the catering recommendation system after making the crossanalysis.
\end{abstract}

\section{Introduction}

Faced with a variety of special diets and lack of nutrition knowledge, people often cannot choose healthy and favorite food. At present, the development of relevant personalized intelligent catering recommendation theory research and application system has gradually become a research hotspot. However, most nutrition recommendation systems focus on the balanced intake of various nutrients elements in Western medicine, which separates the unify and harmony of human's body system. On the contrary, TCM (Traditional Chinese Medicine) dietetic therapy is to grasp the influence of diet on people from the whole body . Therefore, applying the relevant knowledge of TCM constitutional therapeutic theory in the area of healthy catering and menu recommendation for the users, it helps to guide the consumers to rationally plan catering, correct bad eating habits and allow the users not only to enjoy dishes that suit their preferences, but also improve sub-health problems through diet.

* Corresponding author: liuyong@scut.edu.cn 


\section{Analysis of healthy catering recommendation space}

\subsection{Spatial definition}

Define the concepts involved before building a recommended space:

Definition 1 Attribute: The abstract characterization of the object to be described, that is, the relationship between the various properties inherent of a specific object and the interior is called the attribute.

Definition 2 Dimensions: some related attributes of Cartesian subset, which can be expressed as $\mathrm{Di} \subseteq \mathrm{Ai} 1 \times \mathrm{Ai} 2 \times \ldots \times \operatorname{Aij} . \mathrm{Aij}(\mathrm{j}=1, \ldots, \mathrm{k})$ is an attribute of the dimension Di.

Definition 3 Recommended space: $\mathrm{N}$-dimensional classification space to store recommendation information.

\subsection{Attribute analysis}

\subsubsection{Attribute extraction}

The Chinese Medicine Association has published a standard for Table of TCM Constitution Classification and Judgment, which can be used to determine the type of constitution according to the rules. In the catering recommendation, the Decision-Making table allows the system to compute the user's physique through relevant calculations based on the questionnaire submitted by the user. The earliest extant TCM classics in China, Internal Classic Of Huang Di, established the theoretical system of TCM dietetic therapy. According to the above analysis of healthy catering, the attributes of constitution and dieotherapy include two aspects:

(1)Human aspects: constitution, health status, gender, age

(2) Dietotherapy: Five Properties in food, Five Tastes, Channel Tropism, abstinence, Season, Ascending-descending-floating-sinking

\subsubsection{Determine property}

To further identify key attributes, we selected two TCM experts and six college members to conduct interviews. The interview conclusions are as follows:

(1)Dr. Zou of TCM Expert explained that the constitution will be influenced by the geographical location, climate, eating habits, living environment, genetics etc. And these factors will affect a person's physique to varying degrees. (2)Dr. Zhang Lei of TCM Expert stated that dietotherapy is an important part of TCM. For the constitution, food is more to play a nurturing role, rather than the main cause of physical changes in the constitution.

\subsubsection{Property correction}

After the interviews, the initial attributes of healthy catering were revised. The corrected results are shown in Figure 1: 


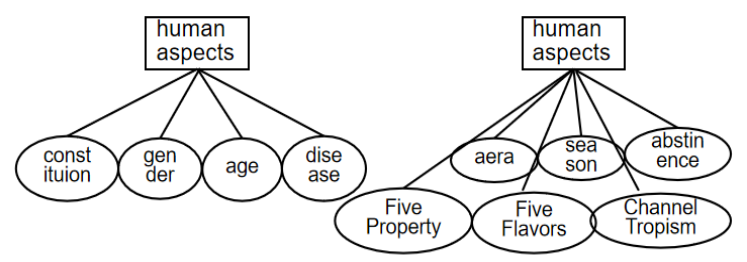

Fig. 1. Healthy catering attributes.

\section{The construction of the recommended space}

\subsection{Spatial dimension determination}

The formal definition of the personalized recommendation system in literature:

Suppose $\mathrm{U}$ is a collection of all users, $\mathrm{I}$ is a collection of all possible items, let $\mathrm{F}$ be the utility function of item $\mathrm{I}$ to user $\mathrm{U}$, that is, $\mathrm{F}: \mathrm{U} \times \mathrm{I} \rightarrow \mathrm{R}$, where $\mathrm{R}$ is a non-negative integer within the specified range or a real number. For any user $u \in U$, it is needed to find a collection of projects $i \in I$ that maximize the utility of user $u$, that is

$$
\forall \mathrm{u} \in \mathrm{U}, \quad I_{u}^{\prime}=\arg \max _{i \in I} F(u, i)
$$

According to the above formula, the recommendation result is based on the user, and the project is recommended content which is calculated based on the matching degree between the user data and the item to be predicted. There is no siutation involved in the traditional recommendation algorithm, but some studies have found that the user's behavior can be affected by the situational information. Users may make different choices in different situations. According to Sandell, up to $40 \%$ of consuming behavior variability can be attributed to Situational Factors, and Personal factors affect only 28 percent of consumer behavior.

\subsection{Construction of dimension attribute}

\subsubsection{User dimension attributes}

User-base attribute contains comprehensive information about the user. According to the knowledge of Constitutional Theory, it can be concluded that gender, age, constitutional type, disease state are the attributes that should be included, as well as height and weight are also issues that must be considered in healthy catering recommendation.

According to the above information, user dimension attributes are summarized as shown in Figure 2.

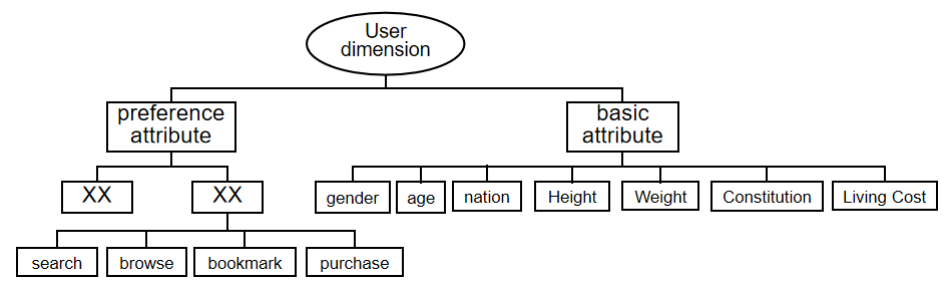

Fig. 2. User dimension attribute. 


\subsubsection{Dish dimension attribute}

In order to study the more characteristics of food, the information is mainly collected from two aspects. On the one hand, the existing catering recommendation platform classifies the dishes, and the other is to summarize based on the existing research materials such as the book literature. Based on the application software research of the food-selling list in the iResearch mobile App index rankings, Lijuan Wang and Yuqiu Liu, have determined the properties of the products for the therapeutic effect of the dishes, as shown in Figure 3.

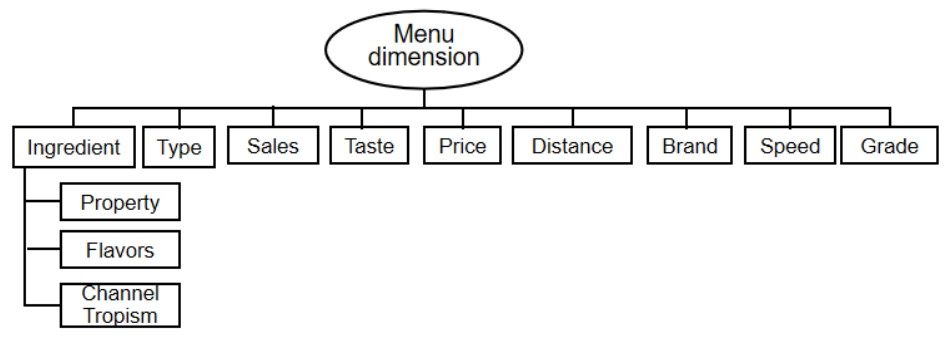

Fig. 3. Menu dimension attribute.

\subsubsection{Situational dimension attribute}

The concept of "situational discovery" was proposed by Google at a conference in 2010. The situational classification method proposed by Professor Junzhong $\mathrm{Gu}$ believes that emphasizing the situation actually reflects that the information recommendation should be transferred from computer-centered to human-centered, and the situation should be considered around the user. Therefore, it summarizes the attributes contained in the situation dimension, as shown in Figure 4.

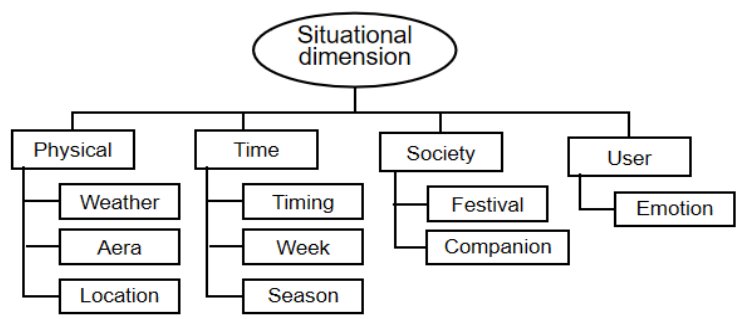

Fig.4. Situational dimension attribute.

\section{Recommended space application analysis}

As the most dynamic consumer group in the Internet economy, college students' consumer groups are selected as the representative of the catering recommendation system research and can be used to verify the validity and importance of their respective attributes.

\subsection{Important result of each attribute}

In the situation attribute, the overall level of the consumer's evaluation of the importance of each attribute is lower than the product attribute, and the difference is also small. The first place, timing, reflects the college students' understanding of the time point when they choose their meal. In the second and third place, moods and dining location, it reflects that people will have different dietary demand under different emotions, and mood has a huge impact on 
dining choices.

\subsection{Cross analysis}

As the level of living expenses increases, the sensitivity to price will become lower and lower. Therefore, for people with higher living expenses, the most important consideration in the choice of food and beverage is the nature of the food itself, and the price has a lower impact on it.

Cross-analysis was carried out between gender and each problem, and the average score of each problem was obtained to draw the score radar chart as shown in figure 5. Therefore, when the system recognizes that the user is male student, more information about improving health status can be pushed. In the design of health and preference weights, the weight of health can also be set higher.

\subsection{Result analysis}

According to the ranking of the importance of each attribute in the survey results and the reasoning of the health status of college students, it can be concluded that the healthy catering recommendation platform based on the theory of dietotherapy has broad market prospects among college students. For the catering platform, the food recommendation should focus on the classification of the taste of dishes and the ingredients used, and analyze the tastes of the users and the types of foods that are preferred in the historical behavior record of the user, and then the food recommendation should refer to the corresponding constitution of the user.

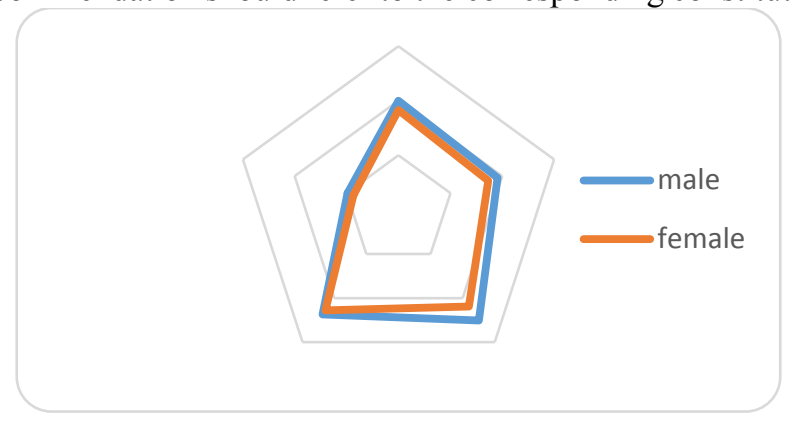

Fig. 5. Radiation map f of health literacy score distribution when gender is different.

This paper was awarded the Innovation and Entrepreneurship Education Reform Research Project of Guangdong (2018A090921).SRP Project of South China University of Technology (X201910561159).

\section{Reference}

1. Sun lihong, He yumin. The difference between Chinese and western nutrition and its enlightenment [J]. Chinese journal of traditional Chinese medicine, 2015(10): 23382340 .

2. Classification and determination of TCM constitution (ZYYXH/T157-2009)[J]. World journal of integrated Chinese and western medicine, 2009(4):303-304.

3. Wang lijuan, Qian yurong. Design and development of personalized menu recommendation system based on Android [J]. Computer knowledge and technology, 2017, 13(20):81-82-100. 
4. Liu yu-qi. Empirical study on factors influencing the purchase intention of online takeaway customers [D]. Southwest jiaotong university, 2017.

5. ALI, Farman, et al. Type-2 fuzzy ontology-aided recommendation systems for IoTbased healthcare[J]. Computer Communications, 2018, 119: 138-155.

6. Subramaniyaswamy V, Manogaran G, Logesh R, et al. An ontology-driven personalized food recommendation in IoT-based healthcare system[J]. Journal of Supercomputing, 2018 (2):1-33.

7. Bennett Rebecca,Zorbas Christina. Prevalence of healthy and unhealthy food and beverage price promotions and their potential influence on shopper purchasing behaviour: A systematic review of the literature.[J]. Obesity reviews, 2019. 